\title{
Effects of Mixed Convection and Navier Slip on a Chemically Reactive Heat and Mass Transfer MHD Fluid Flow Over a Permeable Surface with Convective Boundary Conditions
}

\author{
Fenuga $\mathrm{OJ}^{1 *}$ Safiu MA² and Omowaye AJ \\ ${ }^{1}$ University of Lagos, Akoka, Lagos, Nigeria \\ ${ }^{2}$ Federal University of Technology, Akure, Nigeria
}

\begin{abstract}
The work investigates the effects of mixed convection and Navier slip parameters on a chemically reactive heat and mass MHD fluid flow over a permeable surface with convective boundary conditions. By transforming the system of coupled partial differential equations governing the flow into a system of coupled ordinary differential equations using similarity transformation, the resulting ordinary differential equations are solved using the Fourth order Runge - Kutta method with shooting technique. The effects of mixed convection and Navier parameters on the velocity, temperature and concentration profiles are quantitatively discussed graphically.
\end{abstract}

Keywords: Mixed convection; Navier slip; MHD fluid flow; Convective boundary conditions

\section{Nomenclature}

MHD: Magnetohydrohynomics;

$(\mathrm{x}, \mathrm{y})$ : Coordinate axes or variables;

$(\mathrm{u}, \mathrm{v})$ : Velocity components along the $x$-and $y$-axes;

$\psi$ : Dimensionless stream functions;

g: Acceleration due to gravity;

$\beta_{0}$ : Magnetic Field Strength;

T: Uniform Surface Temperature;

T $\infty$ : Farstream Temperature or temperature at the boundary;

$\mathrm{T}_{\mathrm{f}}$ Temperature at the plate surface;

$\mathrm{u}_{\mathrm{e}}$ : Free Stream Velocity;

C: Concentration;

$\mathrm{C}_{\infty}$ : Far stream concentration or concentration at the boundary;

$\mathrm{C}_{\mathrm{f}}$ : Concentration at the plate surface;

$\mathrm{C}_{\mathrm{p}}$ : Specific heat capacity at constant pressure;

$\gamma$ : Coefficient of Kinematic viscosity;

$\sigma$ : Electrical Conductivity;

$\mathrm{K}_{\mathrm{p}}$ : Permeability of the medium;

K: Thermal conductivity;

Dm: Mass diffusivity;

K: Reaction Rate constant;

$\mathrm{q}_{\mathrm{r}}$ : Radiative heat flux;

$\mathrm{S}_{\mathrm{c}}$ : Schmidt Number;

$\mathrm{P}_{\mathrm{r}}$ :Prandtl number;

M: Magnetic parameter;

Gr: Thermal Grash of number;
$\mathrm{B}_{\mathrm{i}}$ : Biot number;

$\beta c$ : Solutal expansion coefficient;

$\beta_{\tau}$ : Thermal expansion coefficient;

$\lambda$ : Internal heat generation parameter;

Ra: Radiation parameter;

Da: Darcy parameter;

Br: Brinkman number;

Gc: Solutal Grashof number;

Fw: Suction or Injection parameter $(S>0$ for suction and $S<0$ for injection);

$\mathrm{B}_{\mathrm{s}}$ : Convective-diffusion parameter;

$\delta$ : Slip parameter;

$(\theta, \varnothing)$ : Dimensionless temperature and concentration;

Q: Heat release;

$\mathrm{hm}$ : Mass transfer;

hf: Heat transfer

\section{Introduction}

The concept of boundary layer was first introduced by L. Prandtl in 1904 and since then it has been applied to several fluid flow problems.

A boundary layer is formed whenever there is a relative motion

*Corresponding author: Fenuga OJ, Department of Mathematics, University of Lagos, Akoka, Nigeria, Tel: +2348 0550 60122; E-mail: ofenuga@unilag.edu

Received October 01, 2018; Accepted October 27, 2018; Published November 27, 2018

Citation: Fenuga OJ, Safiu MA, Omowaye AJ (2018) Effects of Mixed Convection and Navier Slip on a Chemically Reactive Heat and Mass Transfer MHD Fluid Flow Over a Permeable Surface with Convective Boundary Conditions. J Phys Math 9: 291. doi: 10.4172/2090-0902.1000291

Copyright: ( 2018 Fenuga OJ, et al. This is an open-access article distributed under the terms of the Creative Commons Attribution License, which permits unrestricted use, distribution, and reproduction in any medium, provided the original author and source are credited. 
Citation: Fenuga OJ, Safiu MA, Omowaye AJ (2018) Effects of Mixed Convection and Navier Slip on a Chemically Reactive Heat and Mass Transfer MHD Fluid Flow Over a Permeable Surface with Convective Boundary Conditions. J Phys Math 9: 291. doi: 10.4172/2090-0902.1000291

Page 2 of 9

between the boundary and the fluid. The details of flow within the boundary layer are very important for the understanding of many problems in aerodynamics, including the wind stall, the skin-drag on an object, heat transfers that occur in high speed flight and in naval architecture for the designs of ships and submarines. Bhattacharya et al. [1] studied the slip effects on boundary layer stagnation-point flow and heat transfer towards a shrinking sheet. Chaudary and Kumar [2] studied the stagnation point flow and heat transfer for an electrically conducting fluid over a permeable surface in the presence of a magnetic field where the fluid was acted upon by an external uniform magnetic field and a uniform injection or suction which was directed normal to the plane of the wall. Christian and Yakubu [3] examined the effects of thermal radiation on magneto-hydrodynamic flow over a vertical plate with convective surface boundary condition but not for a chemically reactive fluid. Crane [4] examined the problem of laminar boundary layer flow which arose from the flow of an incompressible viscous fluid past a stretching sheet in which the velocity near the stagnation point is proportional to the distance. Okedayo et al. [5] presented similarity solution to the plane stagnation point flow with convective boundary conditions and obtained global Biot numbers. Adeniyan, Adigun [6] considered the same problem under the influence of a uniform magnetic field which was placed transversely to the direction of the fluid flow. Aziz [7] considered the classical problem of hydrodynamic and thermal boundary layer over a flat plate in a uniform stream of fluid using a similarity solution. Bhattacharya and Gupta [8] established the stability of the mass and heat transfer for the boundary layer over a stretching sheet subject to suction or blowing which had first been looked at by Gupta and Gupta [9]. The same problem was also considered by Mahapatra and Gupta [10] for different stretching and free stream velocities. Ishak [11] considered a steady laminar boundary layer flow over a permeable flat plate in a uniform free stream with bottom surface of the plate heated by convection from a hot fluid using a similarity solution. Jat and Neemawat [12] studied the flow and heat transfer for electrically conducting fluid over a non-linear stretching sheet. The case for a fluid undergoing chemical reaction was considered by Emmanuel et al. [13]. Makinde and Olanrewaju [14] analysed the effects of thermal buoyancy on the Laminar boundary layer about a vertical plate in a uniform stream of fluid under a convective surface boundary condition using Shooting method. Olanrewaju et al. [15] analysed the effects of internal heat generation, thermal radiation and buoyancy force on the Laminar boundary layer about a vertical plate in a uniform stream of fluid under a convective boundary condition with the assumption that the left surface of the plate is in contact with the hot fluid and a stream of cold fluid flow steadily over a right surface; the heat source decays exponentially towards the surface of the plate by using Shooting technique. Fenuga et al. [16] considered the effects of buoyancy force and fluid injection/suction on a chemically reactive MHD flow with Heat and Mass Transfer over a permeable surface in the presence of Heat source/sink. Sajid and Hayat [17] considered the effect of radiation on the boundary layer flow and heat transfer of a viscous fluid over an exponentially stretching sheet using Homotopy Analysis Method. This paper extends the work of Emmanuel et al. [13] together with Fenuga et al. [16] to include mixed convection and Navier slip parameters on a chemically reactive MHD flow with heat and mass transfer over a Permeable surface with convective boundary conditions. The problem will be solved numerically using Runge-Kutta fourth order method with Shooting technique.

\section{Materials and Methods}

Consider a two-dimensional, steady, hydrodynamic boundary layer flow with heat and mass transfer over a permeable uniformly moving plate placed in a saturated porous medium and the plane $y=0$ of a Cartesian coordinates system with the $\mathrm{x}$-axis along the surface of the plate in the presence of an externally applied magnetic field of constant strength $\left(0, \beta_{0}, 0\right)$. The lower surface of the plate is heated by convection from a hot fluid at temperature $\mathrm{T}_{\mathrm{f}}$ to give rise to a coefficient of heat transfer $h_{f}$. Also, the lower surface of the plate is heated by convection from a viscous fluid at concentration $\mathrm{C}_{\mathrm{f}}$ to give rise to a coefficient mass transfer $h_{m}$. Furthermore the upper part of the plate is a Newtonian fluid which is electrically conducting (Figure 1).

Using the baussinesq approximation, the equations governing the fluid flow are:

$$
\begin{aligned}
& \frac{\partial u}{\partial x}+\frac{\partial v}{\partial y}=0 \\
& u \frac{\partial u}{\partial x}+v \frac{\partial u}{\partial y}=\gamma \frac{\partial^{2} u}{\partial y^{2}}+g \beta_{T}\left(T-T_{\infty}\right)-\frac{\sigma \beta_{0}^{2}\left(u-u_{e}\right)}{\rho}-\frac{\gamma}{K_{P}}\left(u-u_{e}\right)+g \beta_{C}\left(C-C_{\infty}\right) \\
& u \frac{\partial T}{\partial x}+v \frac{\partial T}{\partial y}=\frac{k}{\rho C_{P}} \frac{\partial^{2} T}{\partial y^{2}}+\frac{\gamma}{C_{P}}\left(\frac{\partial u}{\partial y}\right)^{2}+\frac{\sigma \beta_{0}^{2}\left(u-u_{e}\right)^{2}}{\rho C_{P}}+\frac{Q}{\rho C_{P}}\left(T-T_{\infty}\right)-\frac{1}{C_{P}} \frac{\partial q_{r}}{\partial y} \\
& u \frac{\partial C}{\partial x}+v \frac{\partial C}{\partial y}=D_{m} \frac{\partial^{2} C}{\partial y^{2}}-K_{r}\left(C-C_{\infty}\right)
\end{aligned}
$$

The corresponding boundary conditions are

$$
\begin{aligned}
& u(x, 0)=L \frac{\partial u}{\partial y}, \quad v(x, 0)= \pm V_{o} \\
& -K \frac{\partial T}{\partial y}(x, 0)=h_{f}\left(T_{f}-T(x, 0)\right) \\
& -D \frac{\partial C}{\partial y}(x, 0)=h_{m}\left(C_{f}-C(x, 0)\right) \\
& \mathrm{u}(\mathrm{x}, \infty)=\mathrm{u}_{\mathrm{e}}, \mathrm{T}(\mathrm{x}, \infty)=\mathrm{T}_{\infty}, \mathrm{C}(\mathrm{x}, \infty)=\mathrm{C}_{\infty}
\end{aligned}
$$

Following Olanrewaju and et al. [18], the similarity variables are

$$
\eta=x^{-\frac{1}{2}} y \sqrt{\frac{u_{e}}{\gamma}}, \text { and } \psi=x^{\frac{1}{2}} \sqrt{\gamma u_{e}} f(\eta)
$$

Where $u=\frac{\partial \psi}{\partial y}, \quad v=-\frac{\partial \psi}{\partial x} ; \quad \theta(\eta)=\frac{T-T_{\infty}}{T_{f}-T_{\infty}}$ and $\varphi(\eta)=\frac{C-C_{\infty}}{C_{f}-C_{\infty}}$

Using the similarity variables, the and that satisfy equation (1) are $u=u_{e} f^{\prime}(\eta)$ and $v=\frac{1}{2} \sqrt{\frac{u_{e} \gamma}{x}}\left(\eta f^{\prime}(\eta)-f(\eta)\right)$

Using eqn. (9) to transforming the momentum, energy and

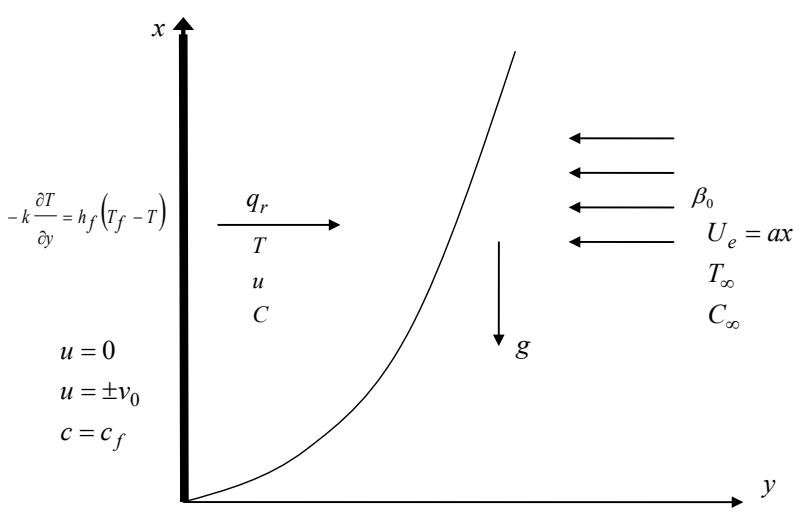

Figure 1: Flow configuration and coordinate system. 
concentration equations (1) to (4) together with the boundary conditions (5) to (9), we obtain

$$
\begin{aligned}
& f^{\prime \prime \prime}+\frac{1}{2} f f^{\prime}+G r_{x} \theta-G c_{x} \varphi+\left(M_{x}+D a_{x}\right)\left(1-f^{\prime}\right)=0 \\
& \left(1+\frac{4}{3} B S_{x} R a\right) \theta^{\prime \prime}+\frac{1}{2} \operatorname{Pr} f \theta^{\prime}+\operatorname{Pr} \lambda_{x} \theta+\operatorname{Pr} E c\left(f^{\prime \prime}\right)^{2}+M_{x} E c \operatorname{Pr}\left(1-f^{\prime}\right)^{2}=0 \\
& \varphi^{\prime \prime}+\frac{1}{2} S_{c} f \varphi^{\prime}-S c \beta_{x} \varphi=0
\end{aligned}
$$

The corresponding boundary conditions are

$$
\begin{aligned}
& f^{\prime}(0)=\delta_{x} f^{\prime \prime}(0) ; f(0)=F_{w x} ; \quad-\theta^{\prime}(0)=B i_{x}(1-\theta(0)) ;-\varphi^{\prime}(0)=B s_{x}(1-\varphi(0)) ; \\
& f^{\prime}(\infty)=1 ; \quad \theta(\infty)=0 ; \quad \varphi(\infty)=0
\end{aligned}
$$

where the prime symbol represents the derivative with respect to $y$,

$S c=\frac{\gamma}{D_{m}}$ is the Schmidt number, $P_{r}=\frac{\gamma}{\alpha}$ is the Prandtl number $M_{x}=\frac{\sigma \beta_{0}^{2} x}{\rho u_{e}}$ is the magnetic parameter, $F_{w x}=\frac{ \pm 2 v_{0} x^{\frac{1}{2}}}{\sqrt{\gamma u_{e}}}$ is the injection/ suction parameter, $G r_{x}=\frac{x g \beta_{\tau}\left(T_{f}-T_{\infty}\right)}{u_{e}^{2}}$ is the thermal Grashof number, $G c_{x}=\frac{x g \beta_{c}\left(C_{f}-C_{\infty}\right)}{u_{e}^{2}}$ is the solutal Grashof number, $\beta_{x}=\frac{k_{r} x}{u_{e}}$ is the reaction rate parameter, $\lambda_{x}=\frac{x Q}{u_{e} \rho c_{p}}$ is the internal heat generation parameter, $R_{a}=\frac{4 \sigma * T_{\infty}^{3}}{k \rho C_{p} \gamma}$ is the radiation parameter, $D a_{x} \frac{x \gamma}{u_{e} k_{p}}$ is the Brinkman number, $B s_{x}=\frac{D}{h_{m}} \sqrt{\frac{u_{e}}{\gamma x}}$ is the convective-diffusion parameter, $B i_{x}=\frac{h_{f}}{k} \sqrt{\frac{\gamma x}{u_{e}}}$ is the Biot number, $\delta_{x}=L \sqrt{\frac{u_{e}}{\gamma x}}$.

It is assumed that equations (10) with boundary conditions (11) have similarity solutions when the parameters $\mathrm{M}_{\mathrm{x}}, \mathrm{F}_{\mathrm{wx}}, \mathrm{Gr}_{\mathrm{x}}, \mathrm{Gc}_{\mathrm{x}}, \beta_{\mathrm{x}}, \lambda_{\mathrm{x}^{\prime}}$ $\mathrm{Da}_{\mathrm{x}}, \mathrm{B}_{\mathrm{Sx}}, \mathrm{Bi}_{\mathrm{x}}$ and $\delta_{\mathrm{x}}$ are defined as constants [19].

\section{Results and Discussion}

Numerical calculations have been carried out for different values of the thermo physical parameters controlling the fluid dynamics in the flow region using Runge-Kutta method with shooting technique implemented by maple 17 (Table 1) illustrates the values of the Skinfriction coefficient $f^{\prime \prime}(0)$, for various values of the embedded flow parameters. Table 2 illustrate the values of the plate surface temperature $\theta(0)$ together with the Nusselt number $-\theta^{\prime}(0)$ for various values of the embedded flow parameters. Table 3 shows the values of the rate of mass transfer $\phi(0)$ and Sherwood number $-\phi^{\prime}(0)$ for various values of embedded flow parameters. Table 4 shows the comparison of Emmanuel et al. with the present work for prandtl number $\mathrm{P}_{\mathrm{r}}=0.72$ and the work is in perfect agreement [20].

Figures 1-6 explains the effects of emerging flow parameters on the velocity profiles. Generally, the fluid velocity is lower at the plate surface and increases gradually to its free stream values satisfying the boundary condition. In Figure 1, as Hartmann number increases, so does the retarding force and hence the velocity profile decreases.

\begin{tabular}{|c|c|c|c|c|c|c|}
\hline $\mathrm{Ha}$ & $\mathrm{Da}$ & $\mathbf{G r}$ & Gc & $F_{w}$ & $\delta$ & $f^{\prime \prime}(0)$ \\
\hline 0.3 & 0.1 & 0.1 & 0.1 & 0.1 & 0.1 & 0.00857 \\
\hline 0.4 & 0.1 & 0.1 & 0.1 & 0.1 & 0.1 & 0.01778 \\
\hline 0.5 & 0.1 & 0.1 & 0.1 & 0.1 & 0.1 & 0.026 \\
\hline 0.1 & 0.5 & 0.1 & 0.1 & 0.1 & 0.1 & 0.87245 \\
\hline 0.1 & 1 & 0.1 & 0.1 & 0.1 & 0.1 & 1.06771 \\
\hline 0.1 & 2 & 0.1 & 0.1 & 0.1 & 0.1 & 1.35479 \\
\hline 0.1 & 0.1 & 0.7 & 0.1 & 0.1 & 0.1 & 0.00185 \\
\hline 0.1 & 0.1 & 0.75 & 0.1 & 0.1 & 0.1 & 0.01771 \\
\hline 0.1 & 0.1 & 0.8 & 0.1 & 0.1 & 0.1 & 0.03663 \\
\hline 0.1 & 0.1 & 0.1 & 0.2 & 0.1 & 0.1 & 2.29323 \\
\hline 0.1 & 0.1 & 0.1 & 0.3 & 0.1 & 0.1 & 2.7965 \\
\hline 0.1 & 0.1 & 0.1 & 0.4 & 0.1 & 0.1 & 3.47131 \\
\hline 0.1 & 0.1 & 0.1 & 0.1 & -0.5 & 0.1 & 0.55816 \\
\hline 0.1 & 0.1 & 0.1 & 0.1 & -0.2 & 0.1 & 0.60042 \\
\hline 0.1 & 0.1 & 0.1 & 0.1 & -0.1 & 0.1 & 0.61897 \\
\hline 0.1 & 0.1 & 0.1 & 0.1 & 0.5 & 0.1 & 0.76119 \\
\hline 0.1 & 0.1 & 0.1 & 0.1 & 0.7 & 0.1 & 0.81654 \\
\hline 0.1 & 0.1 & 0.1 & 0.1 & 3 & 0.1 & 0.90409 \\
\hline 0.1 & 0.1 & 0.1 & 0.1 & 0.1 & 2 & 0.31935 \\
\hline 0.1 & 0.1 & 0.1 & 0.1 & 0.1 & 3 & 0.24789 \\
\hline 0.1 & 0.1 & 0.1 & 0.1 & 0.1 & 5 & 0.17059 \\
\hline
\end{tabular}
Figure 2 shows a decrease in the fluid velocity within the boundary layer due to porosity of the medium thereby decreasing the momentum

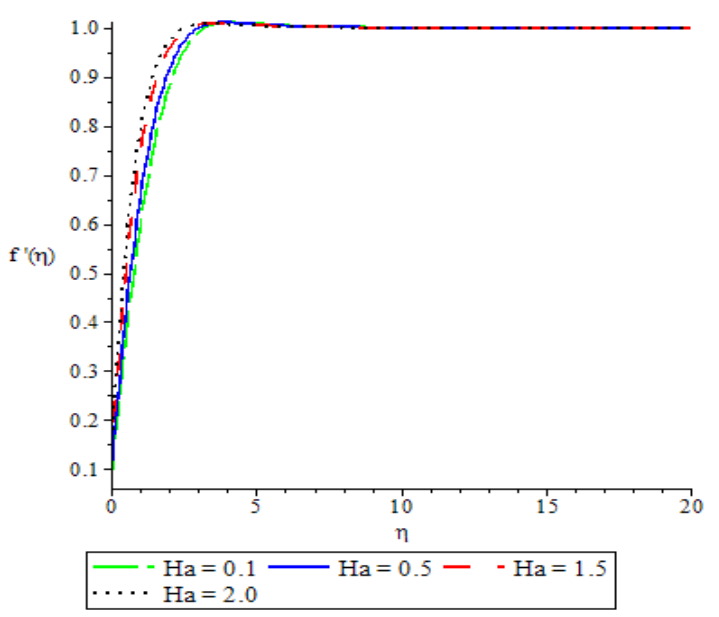

Figure 2: Velocity profiles for varying Ha.

Table 1: Computations showing the values of coefficients of skin-friction with $\mathrm{Ha}, \mathrm{Da}, \mathrm{Gr}, \mathrm{Gc}, \mathrm{F}_{\mathrm{w}}, \delta$ as prescribed parameters. 
Citation: Fenuga OJ, Safiu MA, Omowaye AJ (2018) Effects of Mixed Convection and Navier Slip on a Chemically Reactive Heat and Mass Transfer MHD Fluid Flow Over a Permeable Surface with Convective Boundary Conditions. J Phys Math 9: 291. doi: 10.4172/2090-0902.1000291

Page 4 of 9

\begin{tabular}{|c|c|c|c|c|c|c|c|c|}
\hline Rd & $\mathrm{Br}$ & $\mathrm{Ha}$ & $\mathrm{Pr}$ & $\lambda$ & $\mathrm{Bi}$ & $F_{w}$ & $\theta(0)$ & $-\theta^{\prime}(0)$ \\
\hline 0.5 & 0.1 & 0.1 & 0.72 & 0.2 & 0.1 & 0.1 & 0.46704 & 0.0533 \\
\hline 1 & 0.1 & 0.1 & 0.72 & 0.2 & 0.1 & 0.1 & 0.47016 & 0.05298 \\
\hline 1.5 & 0.1 & 0.1 & 0.72 & 0.2 & 0.1 & 0.1 & 0.47634 & 0.05237 \\
\hline 0.1 & 1.5 & 0.1 & 0.72 & 0.2 & 0.1 & 0.1 & 2.36778 & 0.13678 \\
\hline 0.1 & 1.7 & 0.1 & 0.72 & 0.2 & 0.1 & 0.1 & 2.69636 & 0.16964 \\
\hline 0.1 & 2 & 0.1 & 0.72 & 0.2 & 0.1 & 0.1 & 3.22784 & 0.22278 \\
\hline 0.1 & 0.1 & 8 & 0.72 & 0.2 & 0.1 & 0.1 & 0.6329 & 0.03671 \\
\hline 0.1 & 0.1 & 9 & 0.72 & 0.2 & 0.1 & 0.1 & 0.64146 & 0.03585 \\
\hline 0.1 & 0.1 & 10 & 0.72 & 0.2 & 0.1 & 0.1 & 0.64911 & 0.03509 \\
\hline 0.1 & 0.1 & 0.1 & 1.2 & 0.2 & 0.1 & 0.1 & 0.43898 & 0.0561 \\
\hline 0.1 & 0.1 & 0.1 & 1.7 & 0.2 & 0.1 & 0.1 & 0.42118 & 0.05788 \\
\hline 0.1 & 0.1 & 0.1 & 2 & 0.2 & 0.1 & 0.1 & 0.41469 & 0.05853 \\
\hline 0.1 & 0.1 & 0.1 & 0.1 & 0.05 & 0.1 & 0.1 & 0.31646 & 0.06835 \\
\hline 0.1 & 0.1 & 0.1 & 0.1 & 0.07 & 0.1 & 0.1 & 0.33 & 0.067 \\
\hline 0.1 & 0.1 & 0.1 & 0.1 & 0.1 & 0.1 & 0.1 & 0.35335 & 0.06467 \\
\hline 0.1 & 0.1 & 0.1 & 0.1 & 0.2 & 0.2 & 0.1 & 0.6089 & 0.07822 \\
\hline 0.1 & 0.1 & 0.1 & 0.1 & 0.2 & 0.3 & 0.1 & 0.68795 & 0.09362 \\
\hline 0.1 & 0.1 & 0.1 & 0.1 & 0.2 & 1 & 0.1 & 0.87004 & 0.12996 \\
\hline 0.1 & 0.1 & 0.1 & 0.1 & 0.2 & 0.1 & -0.5 & 0.87786 & 0.01221 \\
\hline 0.1 & 0.1 & 0.1 & 0.1 & 0.2 & 0.1 & -0.2 & 0.62587 & 0.03741 \\
\hline 0.1 & 0.1 & 0.1 & 0.1 & 0.2 & 0.1 & -0.1 & 0.56692 & 0.04331 \\
\hline 0.1 & 0.1 & 0.1 & 0.1 & 0.2 & 0.1 & 0.5 & 0.35461 & 0.06454 \\
\hline 0.1 & 0.1 & 0.1 & 0.1 & 0.2 & 0.1 & 0.7 & 0.31468 & 0.06853 \\
\hline 0.1 & 0.1 & 0.1 & 0.1 & 0.2 & 0.1 & 1 & 0.26956 & 0.07304 \\
\hline
\end{tabular}

Table 2: Computations showing values of the plate temperature $\theta(0)$ and Nusselt number $-\theta^{\prime}(0)$ with $\mathrm{Rd}, \mathrm{Br}, \mathrm{Ha}, \mathrm{Pr}, \lambda, \mathrm{Bi}, \mathrm{F}_{\mathrm{W}}, \theta(0),-\theta^{\prime}(0)$ as prescribed parameters.

\begin{tabular}{|c|c|c|c|c|c|}
\hline Sc & $\beta$ & $F_{w}$ & Bs & $\phi(0)$ & $-\phi^{\prime}(0)$ \\
\hline 0.24 & 0.1 & 0.1 & 0.1 & 0.32577 & 0.06742 \\
\hline 0.64 & 0.1 & 0.1 & 0.1 & 0.24267 & 0.07573 \\
\hline 0.78 & 0.1 & 0.1 & 0.1 & 0.22684 & 0.07732 \\
\hline 0.1 & 0.01 & 0.1 & 0.1 & 0.43841 & 0.05616 \\
\hline 0.1 & 0.05 & 0.1 & 0.1 & 0.41818 & 0.05818 \\
\hline 0.1 & 0.09 & 0.1 & 0.1 & 0.40041 & 0.05996 \\
\hline 0.1 & 0.1 & -0.5 & 0.1 & 0.37328 & 0.06267 \\
\hline 0.1 & 0.1 & -0.2 & 0.1 & 0.36112 & 0.06389 \\
\hline 0.1 & 0.1 & -0.1 & 0.1 & 0.35699 & 0.0643 \\
\hline 0.1 & 0.1 & 0.5 & 0.1 & 0.33277 & 0.06672 \\
\hline 0.1 & 0.1 & 0.7 & 0.1 & 0.32512 & 0.06749 \\
\hline 0.1 & 0.1 & 1 & 0.1 & 0.31413 & 0.06859 \\
\hline 0.1 & 0.1 & 0.1 & 0.3 & 0.66294 & 0.10112 \\
\hline 0.1 & 0.1 & 0.1 & 0.5 & 0.76617 & 0.11692 \\
\hline 0.1 & 0.1 & 0.1 & 1 & 0.86756 & 0.13244 \\
\hline
\end{tabular}

Table 3: Computations showing values of Rate of Mass Transfer $\phi(0)$ and Sherwood number $-\varphi^{\prime}(0)$ with Sc, $\beta, F_{w}$, Bs as prescribed parameters.

\begin{tabular}{|c|c|c|c|c|c|c|c|c|c|c|}
\hline \multirow[b]{2}{*}{$\operatorname{Pr}$} & \multirow[b]{2}{*}{ Sc } & \multirow[b]{2}{*}{ M } & \multirow[b]{2}{*}{$\mathbf{R a}$} & \multirow[b]{2}{*}{$\mathrm{Br}$} & \multirow[b]{2}{*}{$\beta$} & \multirow[b]{2}{*}{$\mathrm{Bi}$} & \multicolumn{2}{|c|}{ Emmanuel et al. [9] } & \multicolumn{2}{|c|}{ Present Paper } \\
\hline & & & & & & & $f^{\prime \prime}(0)$ & $-\theta^{\prime}(0)$ & $f^{\prime \prime}(0)$ & $-\theta^{\prime}(0)$ \\
\hline 0.71 & 0.24 & 0.1 & 0.1 & 0.1 & 0.1 & 0.1 & 0.45184 & 0.06828 & 0.45184 & 0.06828 \\
\hline 0.72 & 0.24 & 0.1 & 0.1 & 0.1 & 0.1 & 0.1 & 0.45184 & 0.06842 & 0.45184 & 0.06842 \\
\hline 0.72 & 0.24 & 0.1 & 0.1 & 0.1 & 0.1 & 0.1 & 0.77079 & 0.06422 & 0.77079 & 0.06422 \\
\hline 0.72 & 0.24 & 0.1 & 0.1 & 0.1 & 0.1 & 0.1 & 0.45184 & 0.06698 & 0.45184 & 0.06698 \\
\hline 0.72 & 0.24 & 0.1 & 0.1 & 0.1 & 0.1 & 0.1 & 0.45184 & 0.04266 & 0.45184 & 0.04266 \\
\hline
\end{tabular}

Table 4: Computations showing comparison of the work with Emmanuel et al. [9] for $n=1$ and $M=R a=B r=\beta=B i=0$. 
boundary layer thickness. In Figure 3, the fluid velocity increases to a more distinctive peak value due to increase in the buoyancy force. Figure 4 shows that an increase in the thermal Grashorf number leads to a thinning in the momentum boundary layer thickness and hence increasing the velocity of the flow. In Figure 5 increase in fluid suction $\mathrm{F}_{\mathrm{w}}>0$ retarded the rate of transport and reduced the boundary layer thickness and an opposite phenomenon was noticed for fluid injection $\mathrm{F}_{\mathrm{w}}>0$ while $\mathrm{F}_{\mathrm{w}}=0$ refers to a non-porous plate. In Figure 6 it was observed that the fluid velocity at the plate surface increases with increase in the Navier slip parameter $\delta$ resulting in the lubrication and slipperiness of the surface [21].

Figure 7 shows the effects of the emerging flow parameter on the temperature profile. The maximum value of the fluid temperature is attained at the plate surface but decreases to the free stream zero value away from the plate satisfying the boundary condition. The thermal boundary layer thickness increases with an increase in Biot number $B_{i}$. This is because as $B_{i}$ increases, the heat transfer rate from the hot fluid at the lower side of the plate to the cold fluid at the upper side increases. The effects of the slip parameter and Prandtl number on the temperature profile are seen in Figures 8 . The temperature profile decreases with increase in slip parameter $\delta$ and Prandtl number $\mathrm{P}_{\mathrm{r}}$ (Figures 9). This is

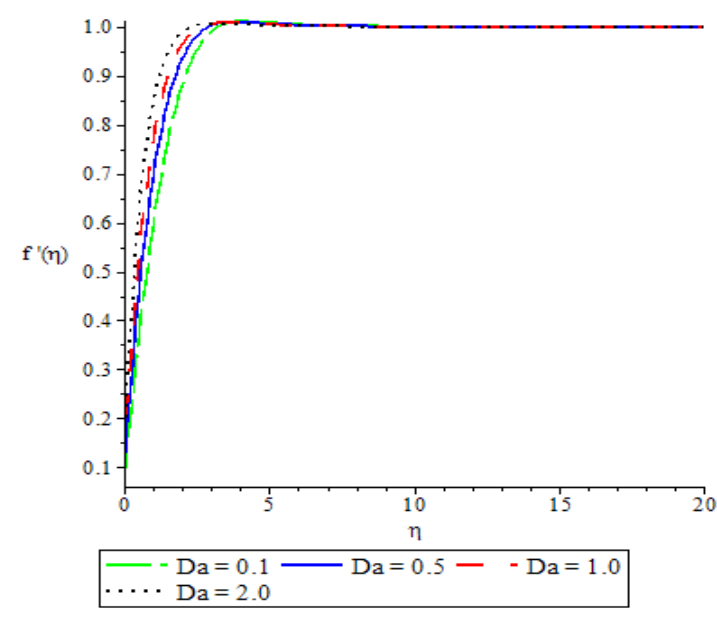

Figure 3: Velocity profiles for varying $\mathrm{Da}$

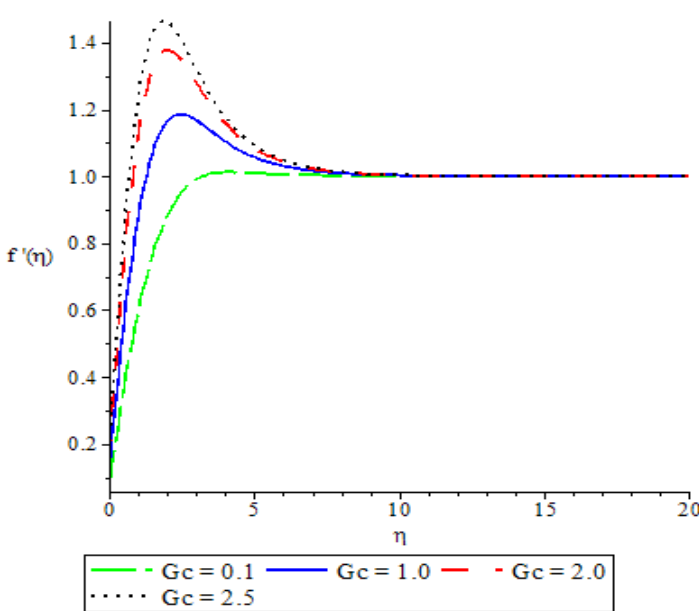

Figure 4: Velocity profiles for varying Gc.

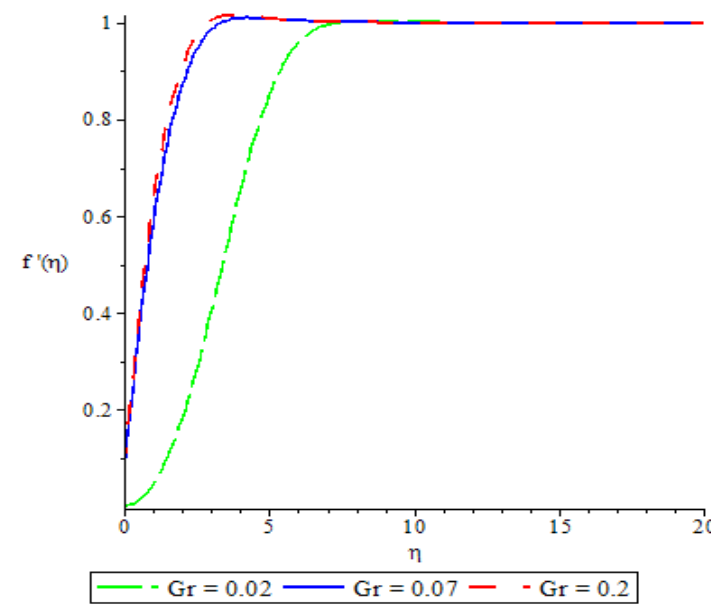

Figure 5: Velocity profiles for varying $\mathrm{Gr}$.

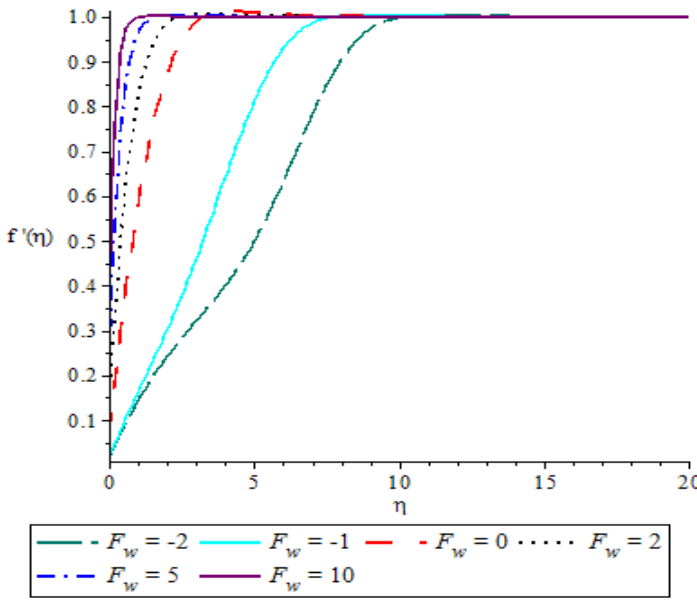

Figure 6: Velocity profiles for varying $F_{w}$.

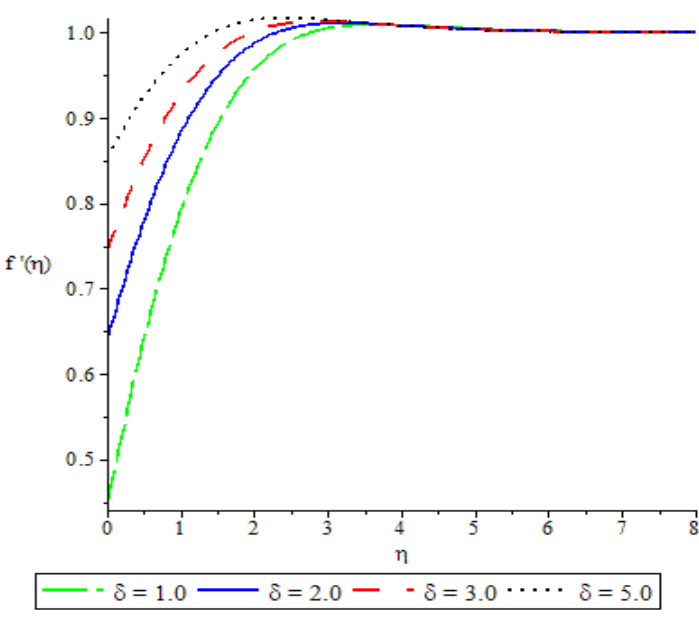

Figure 7: Velocity profiles for varying $\delta$. 


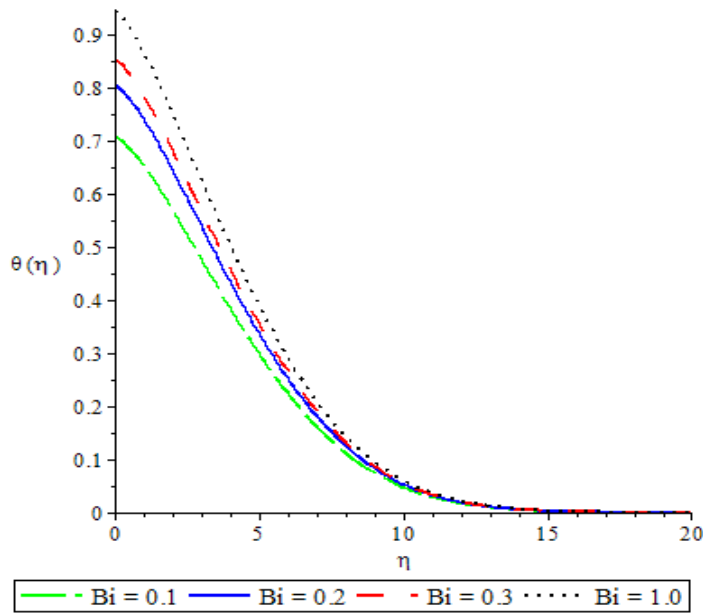

Figure 8: Temperature profiles for varying $\mathrm{Bi}$

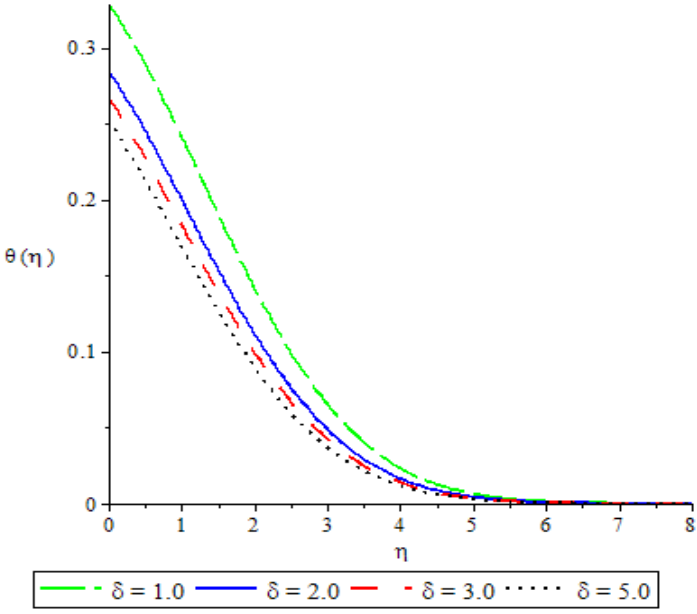

Figure 9: Temperature profiles for varying $\delta$.

because at higher Prandtl number, the fluid has relatively low thermal conductivity, which reduces conduction together with the thermal boundary layer thickness and thereby decreasing the temperature. Figure 10 shows the graph of temperature against the coordinate $\mathrm{n}$ for various values of radiation parameter. Due to absorption, the thermal boundary layer thickness increases as the radiation parameter $\mathrm{Ra}$ increases. Also, in Figure 11, increase in the magnetic parameter $\mathrm{M}$ increases the fluid temperature which in turn, increases the thermal boundary layer thickness due to ohmic heating on the flow system. In Figure 12, progressive rise in fluid temperature and thickening of the thermal boundary layer were observed phenomena as Brinkman number $B_{r}$ and internal heat generation parameter $\lambda$ increases. Fluid suction was found to make the thermal boundary layer thinner as shown in Figures 13.

Figure 14 exhibit the variations of concentration profile against dimensionless variable $\eta$ under the influence of chemical reaction rate parameter $\beta$, Schmidt number Sc, convective-diffusion parameter $\mathrm{B}_{\mathrm{S}}$ and fluid Suction/Injection $\mathrm{F}_{\mathrm{w}}$ (Figures 15). In Figures 15 and 16 , concentration decreases with increase in chemical reaction parameter $\beta$ and fluid Injection $\mathrm{F}_{\mathrm{w}}>0$ but increases with increase in the convective-diffusion parameter $\mathrm{B}_{\mathrm{s}}$ (Figures 16). The solutal boundary

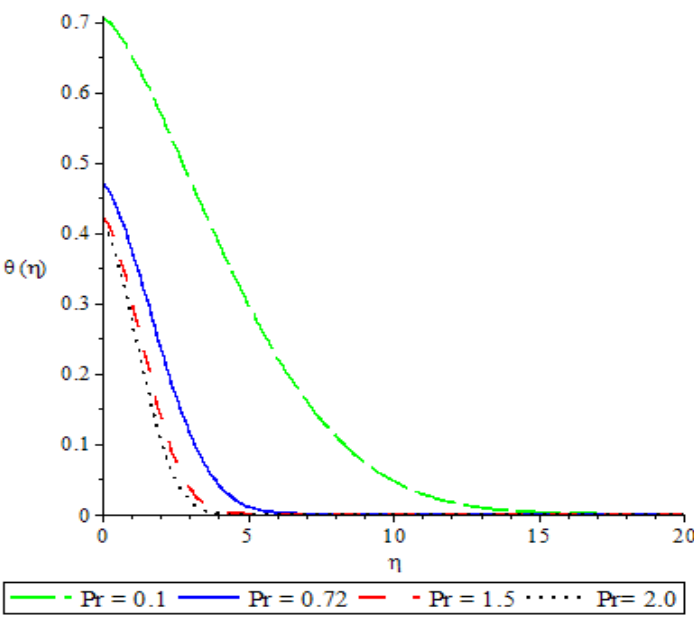

Figure 10: Temperature profiles for varying Pr.

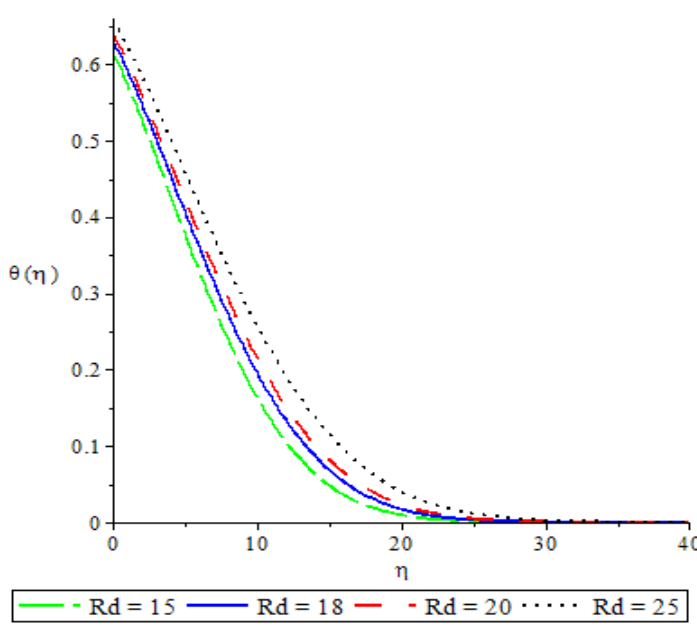

Figure 11: Temperature profiles for varying $\mathrm{Rd}$.

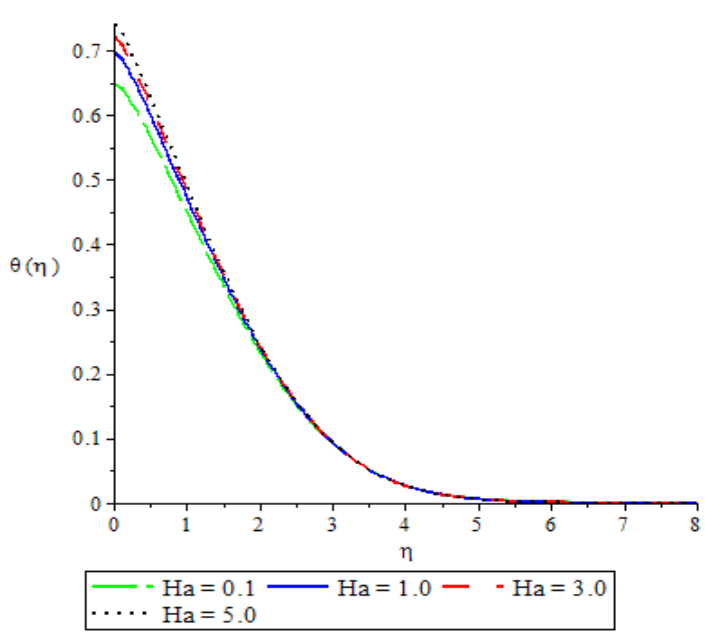

Figure 12: Temperature profiles for varying $\mathrm{Ha}$. 


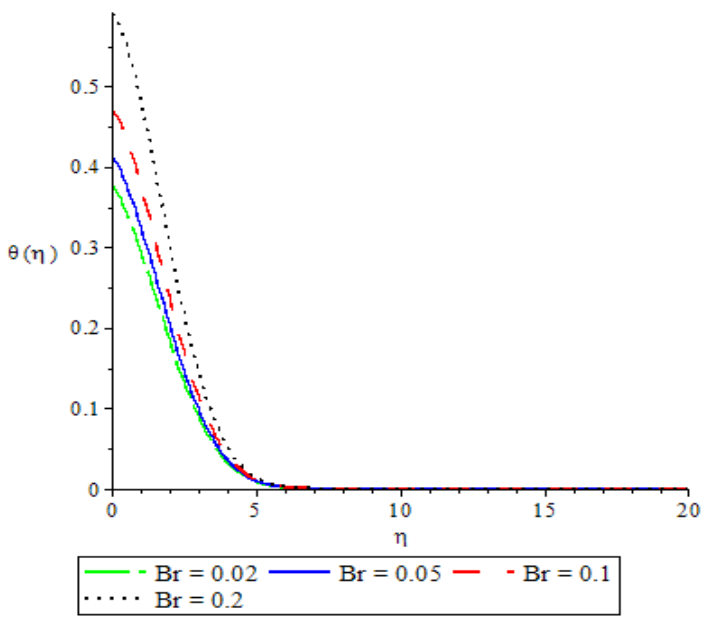

Figure 13: Temperature profiles for varying $\mathrm{Br}$.

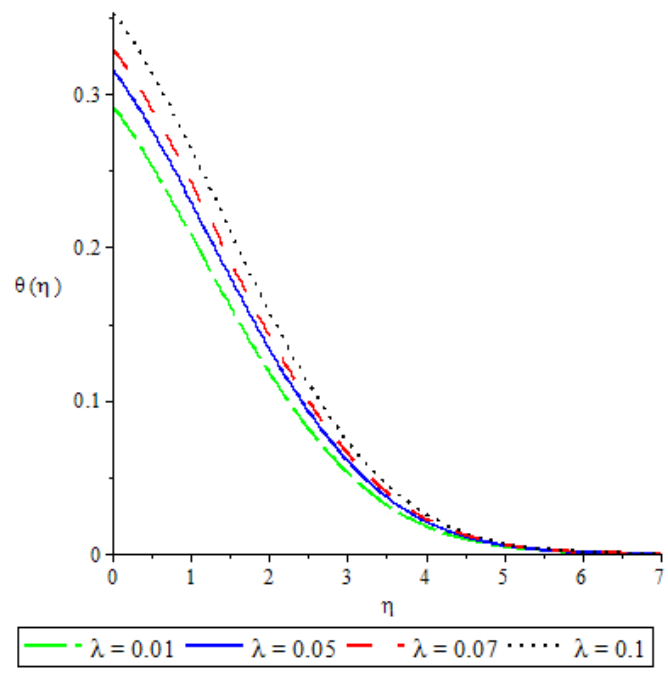

Figure 14: Temperature profiles for varying $\lambda$

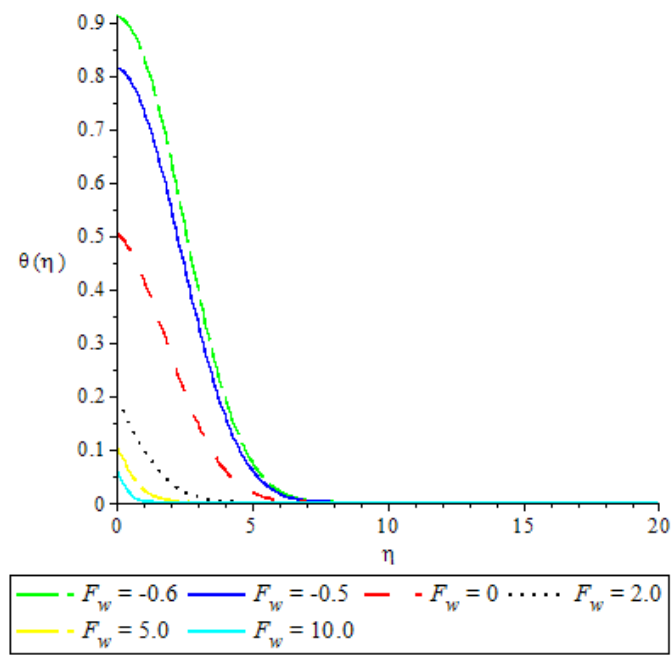

Figure 15: Temperature profiles for varying $F_{w}$. layer thickness was also seen to decrease for appreciable values of $B_{s}$ (Figure 17). The plate surface concentration was increased as a result of increase in $B_{S}$ but reduced for increase in the values of $S c, \beta$ and $F_{w}$. It was observed from Figure 18 that the concentration level of the fluid drops due to increasing chemical reaction parameter, fluid injection and Schmidt number because mass diffusivity raises the concentration level steadily (Figure 19) [22].

\section{Conclusions}

From the numerical solutions and graphical representations, we can conclude that:

- The fluid velocity decreases at the plate and increases gradually to its free stream satisfying the boundary condition.

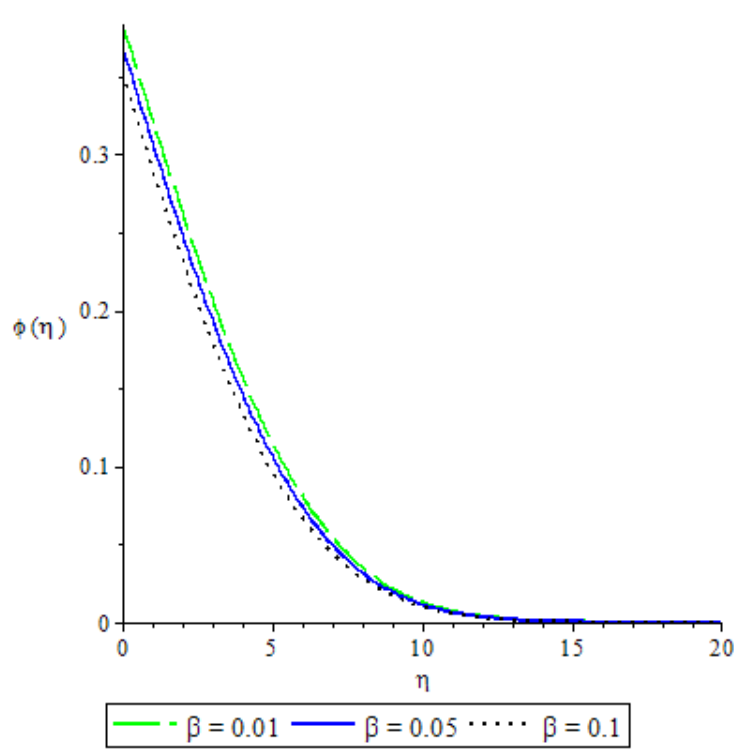

Figure 16: Concentration profiles for varying $\beta$.

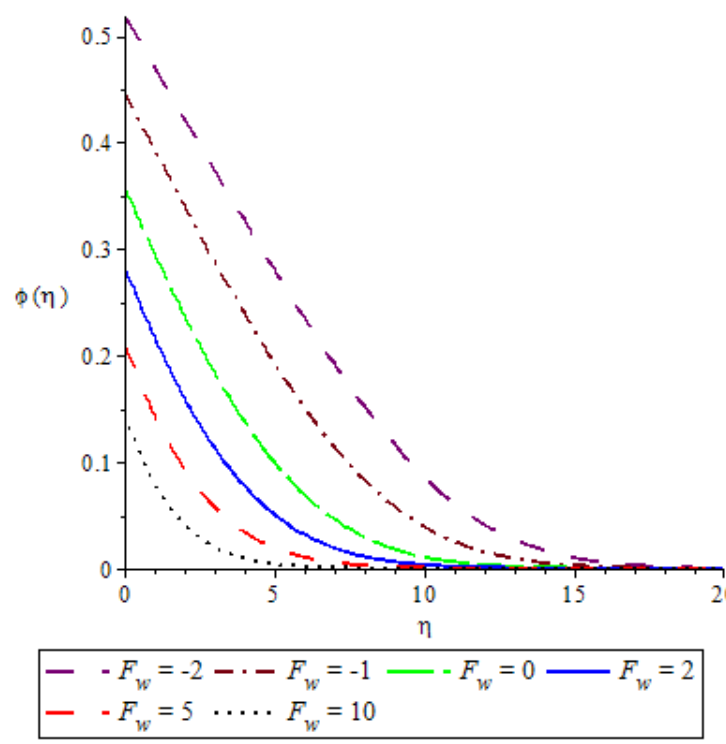

Figure 17: Concentration profiles for varying $F_{w}$. 


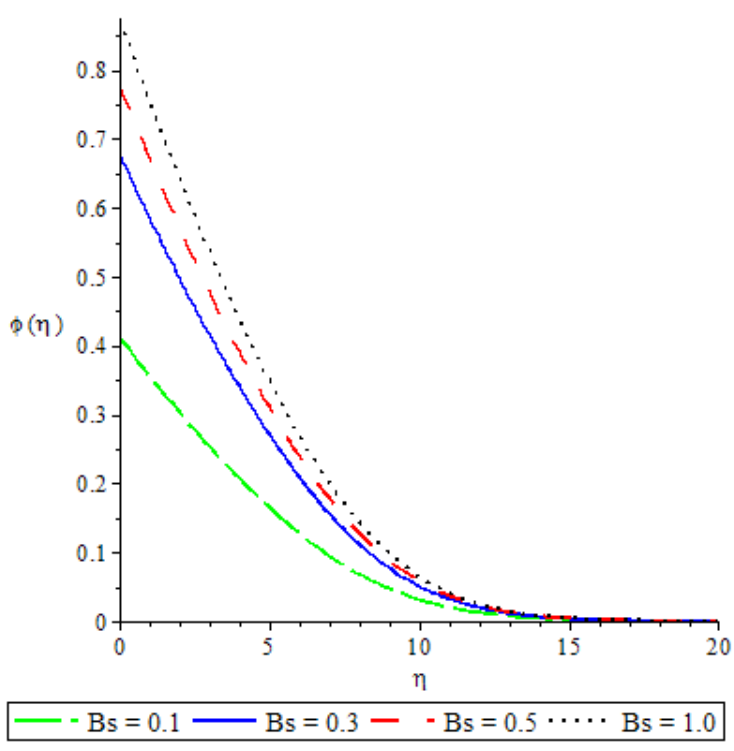

Figure 18: Concentration profiles for varying Bs

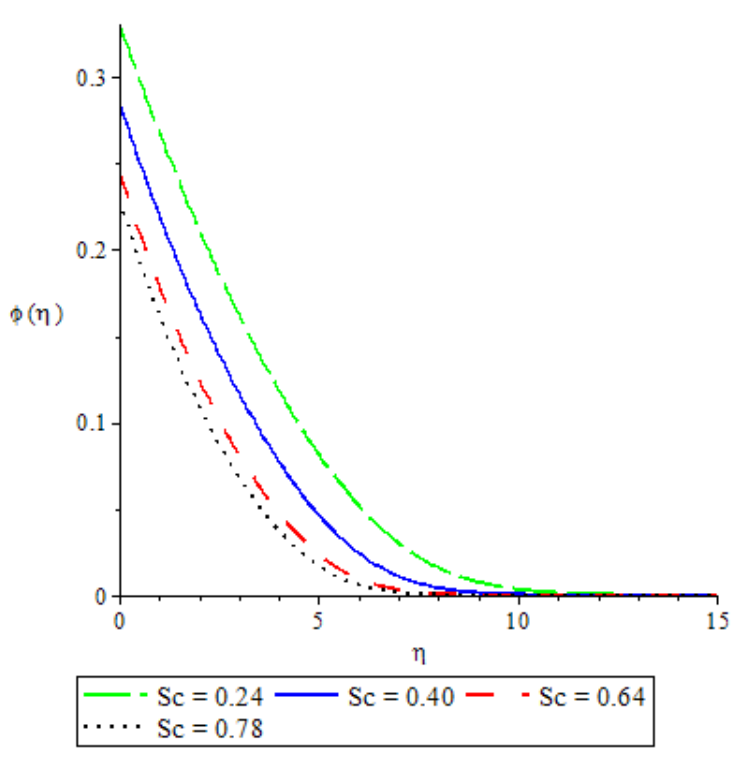

Figure 19: Concentration profiles for varying Sc.

- The fluid velocity at plate surface increases with increases in Navieer slip parameter resulting in the lubrication and slipperiness of the surface.

- The maximum value of the fluid temperature is attained at the plate surface but decreases to the free stream value of zero away from the plate satisfying the boundary condition.

- The fluid temperature decreases with increase in Navier slip parameter and prandtl number due to relatively low conductivity.

- Fluid temperature and the thickening of the thermal boundary layer increases as Brickman number $\mathrm{Br}$ and internal heat generation parameter $\lambda$ increases.
- Thermal boundary layer thickness increases with increase in biot numbers and fluid suction makes the boundary layer thinner.

- The rate of heat transfer (from the plate to the fluid) decreases with thermal radiation and heat source parameter but increases with increase in Brinkman number, Hartmann number, Pandt number and ohmic heating of the flow system.

- Concentration of the flow decreases with increase in chemical reaction $\beta_{x}$, Schmidt number Sc, suction parameter Fw and convective-diffusion parameter $\mathrm{B}_{\mathrm{s}}$.

\section{Acknowledgement} paper.

We appreciate the comments of the reviewers in improving the quality of the

\section{References}

1. Bhattachaya K, Mukhopadyay S, Layek GC (2011) Slip effects of boundary layer stagnation point flow and heat transfer towards a shrinking sheet. Int Jo Heat Mass Transf 54: 308-313.

2. Chaudary S, Kumar P (2013) MHD stagnation point flow and heat transfer over a permeable surface. J Sci Res 15: 50-55.

3. Christian JE, Yakubu IS (2014) Radiative MHD flow over a vertical plate with convective boundary condition. Am J Math 2(6): 214-220.

4. Crane LJ (1970) Flow past a stretching plate. Zeitschrift fiir Angewandte, Math P 21: 645-647.

5. Okedayo TG, Olanrewaju PO, Gbadeyan JA (2012) Analysis of convective plane stagnation point flow with convective boundary conditions. Int $\mathrm{J} \mathrm{Sc}$ Technol 12: 15-17

6. Adeniyan A, Adigun JA (2013) Studies of the effects of convective stagnation point MHD flow with convective boundary conditions in the presence of a uniform magnetic field. Int J Eng Sci 2: 310-313.

7. Aziz A (2009) A similarity solution for Laminar boundary layer over a flat plate with a convective surface boundary condition. Commun of Nonlinear sciences and Numer simulation. 14: 1064-1068.

8. Bhattacharyan SN, Gupta AS (1985) The stability of viscous flow over a stretching sheet. Q Appl Math 43: 359-367.

9. Gupta PS, Gupta AS (1977) Heat and Mass transfer on a stretching sheet with suction or blowing, Canadian J Chem Eng 55: 744-746.

10. Mahapatra TR, Gupta AS (2002) Heat transfer in stagnation point flow towards a stretching sheet. Heat mass transf Spinger 38: 517-521.

11. Ishak $A$ (2010) Similarity solutions for flow and heat transfer over a permeable surface with convective boundary conditions. Appl Math comput 217: 837-842.

12. Jat RN, Abhishak N (2012) Similarity solution for MHD stagnation point flow and heat transfer over a non-linear stretching sheet. Int J Recent Res Rev 13 $32-51$

13. Emmanuel MA, Ibrahim YS, Aziz A (2014) Chemically Reacting Hydromagnetic flow over a Flat surface in the presence of Radiation with viscous Disipation and convective Boundary conditions. Am J Appl Math 2: 179-185.

14. Makinde OD, Olanrewaju PO (2010) Buoyancy Effects on Thermal Boundary layer over a vertical plate with a convective surface Boundary condition. J Fluid Eng 132: 1-4.

15. Olanrewaju PO, Gbadeyan JA, Hayat T, Hendi AA (2011) Effects of Interna heat generation, thermal radiation and buoyancy force on a boundary layer over a vertical plate with a convective surface boundary condition. S Afr J Sci 107: 1-6.

16. Fenuga OJ, Adigun JA, Hassan AR, Olanrewaju PO (2015) Comments on the effects of Buoyancy force and fluid Injection/ Suction on a Chemically reactive MHD flow with Heat and Mass Transfer over a permeable surface in the presence of Heat source/sink. Int J Sci Eng Res 6: 1041-1051.

17. Sajid M, Hayat $T$ (2008) Influence of thermal radiation on the boundary layer flow due to an exponential stretching sheet. Science Direct, Elsevier, 35: 347 356 
Citation: Fenuga OJ, Safiu MA, Omowaye AJ (2018) Effects of Mixed Convection and Navier Slip on a Chemically Reactive Heat and Mass Transfer MHD Fluid Flow Over a Permeable Surface with Convective Boundary Conditions. J Phys Math 9: 291. doi: 10.4172/2090-0902.1000291

Page 9 of 9

18. Conte SD, Boor C (1981) Elementary Numerical Analysis (3rdedn), MC GrawHill Book Co, New York, p: 432.

19. Heck A (2003) Introduction to Maple (3rdedn), Springer-verlag, pp: 1-7.

20. Jain MK, lyaengar SR, Jain RK (1985) Numerical Methods for Scientific and Engineering Computation. Wiley Eastern Ltd, New Delhi, India, p: 406.
21. Jain M (1984) Numerical Solution of Differential Equations, Wiley Eastern Ltd, New Delhi, India, p: 286

22. Krishnamurthy EV, Sen SK (1986) Numerical Algorithms. Affiliated East-West Pvt. Ltd. New Delhi, India. 